\title{
VARIABILIDADE DA FREQUÊNCIA CARDÍACA EM CRIANÇAS OBESAS
}

\author{
HEART RATE VARIABILITY IN OBESE CHILDREN
}

\author{
Naiara Maria de Souza ${ }^{1}$, Renata Claudino Rossi ${ }^{2}$, Franciele Marques Vanderlei ${ }^{3}$, \\ Ana Laura Ricci Vitor ${ }^{1}$, Aline Fernanda Barbosa Bernardo ${ }^{1}$, \\ Ana Clara Campagnolo Real Gonçalves ${ }^{2}$, Lucas Lima Ferreira ${ }^{1}$, Luiz Carlos Marques Vanderlei ${ }^{4}$
}

\section{RESUMO:}

Introdução: nos últimos anos, diversos estudos analisaram o comportamento do sistema nervoso autônomo (SNA) frente à obesidade e utilizaram, para esta análise, a variabilidade da frequência cardíaca (VFC). Embora descrito na literatura que adultos obesos apresentam disfunções do SNA, faltam informações referentes a crianças obesas e os achados existentes ainda são inconclusivos. Objetivo: reunir estudos que abordem a análise do SNA, por meio da VFC, em crianças obesas para melhor compreender a influência da obesidade sobre esse sistema nessa população e fornecer uma atualização dos achados publicados nos últimos onze anos referentes ao tema. Método: a busca dos artigos foi realizada nas bases de dados Pubmed, Ibecs, Medline, Scielo, Cochrane e Lilacs por meio dos seguintes descritores da área da saúde (DeCS): frequência cardíaca, sistema nervoso autônomo, obesidade, criança e sistema nervoso simpático e suas respectivas versões na língua inglesa (MeSH). Resultados: a busca resultou em 11 artigos, os quais demonstraram que, em geral, crianças obesas apresentam menor atividade do SNA parassimpático e os resultados são inconclusivos para a atividade simpática. Além disso, intervenções como dieta e atividade física podem melhorar a condição de atuação do SNA nessas crianças. Conclusão: crianças obesas apresentam modificações na atuação do SNA, o que demonstra a necessidade de atenção precoce a esta população para evitar complicações futuras.

Palavras-chave: criança; obesidade; sistema nervoso autônomo; sistema nervoso parassimpático; frequência cardíaca.

\section{ABSTRACT:}

Introduction: in recent years, several studies have performed the behavior of ANS on obesity by the heart rate variability (HRV). The literature describes that obese adults have dysfunctions on ANS, nevertheless there is a few information with childhood obesity and the findings are still inconclusive. Objective: gathering studies that approach the analysis of the ANS by HRV in obese children to understand better the influence of obesity on this system and in this population and provide an update of the findings published in the last eleven years. Methods: the search of articles was conducted on Pubmed, Ibesc, Medline, Scielo, Cochrane and Lilacs databases using the keywords: heart rate, autonomic nervous system, obesity, child and sympathetic nervous system of health subject headings (MeSH). Results: the electronic search resulted in 11 articles and, in general, showed a decrease in activity of the parasympathetic branch of obese children and inconclusive results by the sympathetic branch. Moreover, interventions as diet and physical activity could increase the ANS function in these children. Conclusion: obese children have changes on ANS function and this presents the necessity of precocious attention aims at avoiding future complications.

Key words: child; obesity; autonomic nervous system; parasympathetic autonomic nervous system; heart rate; review.

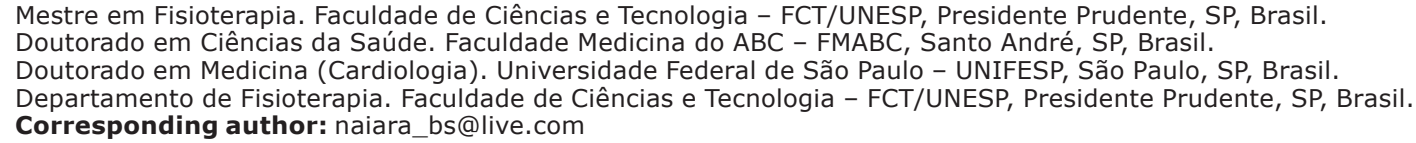




\section{INTRODUÇÃO}

Dos transtornos nutricionais na infância, a obesidade - definida como a deposição e armazenamento excessivo de gordura no organismo, causada geralmente pela interação de diversos fatores, dentre eles maus hábitos alimentares, genética, fatores emocionais, sexo, idade, nível de atividade física e aspectos culturais ${ }^{1-3}$ - é considerada um importante problema de saúde pública ${ }^{4}$.

Dados epidemiológicos mostram que a obesidade vem aumentando significantemente nos últimos anos e segundo a Organização Mundial da Saúde afeta, atualmente, 35\% da população mundial. No Brasil, cerca de $24 \%$ da população estão acima do peso esperado e entre os jovens a obesidade, nos últimos anos, aumentou de 3,7\% para $12,6 \% 5,6$. Em relação às crianças brasileiras, dados preliminares mostram que $7 \%$ estão em situação de excesso de peso, variando de $6 \%$ na região Norte a $9 \%$ na região Sul, indicando exposição moderada à obesidade em todas as regiões do país?.

As consequências da obesidade incluem, dentre outros, distúrbios metabólicos, endócrinos e cardiovasculares, como hipertensão arterial, arteriosclerose e arritmias graves ${ }^{8-10}$. Quando presente na infância ou na adolescência, a obesidade está associada a maior ocorrência de disfunções cardiovasculares e metabólicas na idade adulta ${ }^{11}$. Além disso, estudos têm demonstrado que a obesidade produz alterações na função do sistema nervoso autônomo (SNA) 9,12-15, o que representa uma condição importante, já que esse sistema controla parte das funções internas do corpo.

Nos últimos anos, diversos estudos analisaram o comportamento do SNA frente à obesidade ${ }^{16-19}$ e utilizaram para esta análise a variabilidade da frequência cardíaca (VFC), uma medida não invasiva dos impulsos autonômicos que indica a habilidade do coração em responder aos estímulos fisiológicos, a qual pode ser utilizada em diversas condições, inclusive patológicas ${ }^{20-22}$.

Embora descrito na literatura que adultos obesos apresentam disfunções do SNA ${ }^{1,23,24}$, faltam informações referentes a crianças obesas e os achados existentes ainda são inconclusivos s,25,26. $^{8}$.

Neste contexto e diante da importância em identificar precocemente as alterações induzidas pela obesidade no SNA, buscou-se reunir estudos que abordassem a análise do SNA por meio da VFC em crianças obesas para melhor compreender a influência da obesidade sobre esse sistema nessa população e fornecer uma atualização dos achados publicados nos últimos onze anos referentes ao tema. Estudos que envolvam essas questões podem fornecer informações importantes, de modo que ações precoces e direcionadas a melhorar as condições desse sistema possam ser aplicadas nessa população.

Assim, o objetivo é verificar os efeitos da obesidade no comportamento do sistema nervoso autônomo em crianças.

\section{MÉTODO}

Os artigos utilizados nesse estudo foram selecionados em fevereiro de 2011 e as bases de dados Pubmed, Ibecs, Medline, Scielo, Cochrane e Lilacs foram utilizadas para essa finalidade. Para esta busca, foram utilizados os cruzamentos das palavraschave: frequência cardíaca, sistema nervoso autônomo, obesidade, criança e sistema nervoso simpático, as quais foram definidas com base nos descritores da área da saúde (DeCS) e seus respectivos correspondentes na língua inglesa (MeSH).

Os estudos passaram, inicialmente, por uma seleção com base em seus títulos e foram analisados por um único pesquisador, que os selecionou de acordo com os critérios de inclusão estabelecidos para esta revisão. Para tanto, o titulo deveria expressar como foco do estudo: a VFC em crianças obesas, a influência da obesidade sobre o SNA, as consequências da obesidade na saúde de crianças e aqueles que relacionavam a obesidade ou alguma informação referente a esse quadro, como gordura corporal, com as palavras sistema nervoso parassimpático e/ou simpático, alterações hemodinâmicas e metabólicas. Após isso, foi feita uma filtragem dos resultados para identificação das repetições, já que a busca foi realizada em diversas bases de dados.

Após essa triagem, todos os títulos escolhidos tiveram seus resumos estudados detalhadamente com o objetivo de selecionar os artigos que abordassem a VFC em crianças obesas. Sequencialmente, os resumos que abordassem essa temática tiveram seus textos completos lidos integralmente.

Além disso, todos os estudos selecionados tiveram suas referências analisadas de forma independente, para identificação de estudos relevantes que não foram encontrados na busca eletrônica. Todas as etapas foram acompanhadas por um revisor sênior que realizou o julgamento final de inclusão dos artigos.

Foram inclusos estudos publicados entre o período de 2000 a 2011 nos idiomas português, espanhol e inglês, com crianças obesas de até 12 anos que analisassem o SNA por meio da VFC. Foram inclusos todos os tipos de desenho de estudo.

Os dados foram descritos de forma qualitativa e tabulados de acordo com os autores e ano do estudo, características clinicas e físicas da população, objetivo do trabalho, índices avaliados e conclusões observadas.

\section{RESULTADOS}

\section{Estratégia de busca e seleção}

Por meio da utilização dos descritores na busca das bases de dados, foram encontrados 3128 títulos. A primeira seleção resultou na eliminação de 2829 títulos, e os estudos restantes passaram para a próxima fase da seleção, a qual analisou o conteúdo dos seus resumos e eliminou 170 resu- 
mos, os quais não atenderam aos critérios previamente estabelecidos. Os 129 textos restantes tiveram seus trabalhos lidos integralmente e após a última etapa de eliminação, a qual excluiu um total de 118 estudos, resultaram 11 artigos.

\section{Características dos estudos selecionados}

A tabela 01, organizada por autores e ano de publicação, características da população, objetivos, índices avaliados e conclusões, contém os 11 artigos resultantes da busca sistemática.

Tabela 1: Descrição dos estudos de acordo com seus autores, ano de publicação, características da população, objetivos, índices avaliados e conclusões

\begin{tabular}{|c|c|c|c|c|}
\hline $\begin{array}{l}\text { Autores e ano } \\
\text { de publicação }\end{array}$ & Características da população & Objetivos & Índices avaliados & Conclusões \\
\hline $\begin{array}{l}\text { Nagai et al., } \\
2003 .\end{array}$ & $\begin{array}{l}\text { Grupo obeso }(\mathrm{N}=42) \text { : Média de } \\
\text { idade de } 9,0 \pm 0,3 \text { anos e IMC } \\
23,4 \pm 0,5 \mathrm{Kg} / \mathrm{m}^{2} \text {. } \\
\text { Grupo não obeso }(\mathrm{N}=42) \text { : Média } \\
\text { de idade de } 9,0 \pm 0,3 \text { anos e IMC } \\
17,9 \pm 0,6 \mathrm{Kg} / \mathrm{m}^{2} \text {. }\end{array}$ & $\begin{array}{l}\text { Investigar se o SNS e/ou } \\
\text { SNP estão alterados em } \\
\text { crianças obesas e se há cor- } \\
\text { relação da duração da obe- } \\
\text { sidade com o grau de dis- } \\
\text { função do SNA. }\end{array}$ & $\begin{array}{l}\operatorname{LF}\left(\mathrm{ms}^{2}\right)^{*}, \mathrm{HF}\left(\mathrm{ms}^{2}\right)^{*} \\
\operatorname{eTP}\left(\mathrm{ms}^{2}\right)^{*}\end{array}$ & $\begin{array}{l}\text { Ambos os ramos do SNA sofrem influência } \\
\text { da obesidade, apresentando menor ativi- } \\
\text { dade desse sistema. Além disso, foi possí- } \\
\text { vel constatar uma correlação negativa da } \\
\text { duração da obesidade com os valores de } \\
\text { LF, HF e TP, indicando que a duração da } \\
\text { obesidade influencia o quadro de disfun- } \\
\text { ção do SNA. }\end{array}$ \\
\hline $\begin{array}{l}\text { Nagai et al., } \\
2004 .\end{array}$ & $\begin{array}{l}\text { Grupo obeso ativo }(\mathrm{N}=24) \text { : } \\
\text { Média de idade de } 9,5 \pm 1,4 \text { anos, } \\
\text { IMC } 23,0 \pm 2,5 \mathrm{Kg} / \mathrm{m}^{2} \text {. } \\
\text { Grupo obeso inativo }(\mathrm{N}=24) \text { : } \\
\text { Média de idade de } 9,3 \pm 1,7 \text { anos, } \\
\text { IMC } 24,3 \pm 3,4 \mathrm{Kg} / \mathrm{m}^{2} \text {. } \\
\text { Grupo não obeso ativo }(\mathrm{N}=24) \text { : } \\
\text { Média de idade de } 9,6 \pm 1,3 \text { anos, } \\
\text { IMC } 17,0 \pm 1,4 \mathrm{Kg} / \mathrm{m}^{2} \text {. } \\
\text { Grupo não obeso inativo }(\mathrm{N}= \\
\text { 24): Média de idade de } 9,4 \pm 1,8 \\
\text { anos, IMC } 17,0 \pm 1,5 \mathrm{Kg} / \mathrm{m}^{2} .\end{array}$ & $\begin{array}{l}\text { Avaliar o SNA por meio da } \\
\text { VFC de crianças obesas e } \\
\text { não obesas, as quais foram } \\
\text { divididas em ativos e inati- } \\
\text { vos, para verificar se os SNS } \\
\text { e SNP estão alterados devi- } \\
\text { do a obesidade e a inativi- } \\
\text { dade física. }\end{array}$ & $\begin{array}{l}\operatorname{LF}\left(m s^{2}\right)^{*}, \operatorname{HF}\left(m s^{2}\right)^{*} \\
\text { e } \operatorname{TP}\left(\mathrm{ms}^{2}\right)^{*}\end{array}$ & $\begin{array}{l}\text { Crianças obesas apresentam menores va- } \\
\text { lores de LF, HF e TP em relação a crianças } \\
\text { não obesas, as quais apresentam níveis de } \\
\text { atividade física semelhantes. Portanto os } \\
\text { SNS e SNP de crianças obesas apresentam } \\
\text { disfunções. }\end{array}$ \\
\hline $\begin{array}{l}\text { Kaufman et al., } \\
2007 .\end{array}$ & $\begin{array}{l}\text { Grupo obeso }(\mathrm{N}=10) \text { : Média de } \\
\text { idade } 11,5 \pm 0,8 \text { anos e IMC } 32,4 \\
\pm 5,5 \mathrm{Kg} / \mathrm{m}^{2} \text {. } \\
\text { Grupo acima do peso }(\mathrm{N}=10) \text { : } \\
\text { Média de idade } 11,3 \pm 0,5 \text { anos e } \\
\text { IMC } 24,8 \pm 1,5 \mathrm{Kg} / \mathrm{m}^{2} \text {. } \\
\text { Grupo não obeso }(\mathrm{N}=10) \text { : Média } \\
\text { de idade } 11,5 \pm 1,1 \text { anos e IMC } \\
18,1 \pm 3,1 \mathrm{Kg} / \mathrm{m}^{2} \text {. }\end{array}$ & $\begin{array}{l}\text { Examinar a função autonô- } \\
\text { mica cardiovascular e sua } \\
\text { potencial relação com resis- } \\
\text { tência a leptina, resistência a } \\
\text { insulina, estresse oxidativo e } \\
\text { inflamatório em uma amos- } \\
\text { tra de crianças com diferen- } \\
\text { tes níveis de obesidade. }\end{array}$ & $\begin{array}{l}\text { Média dos Intervalos } \\
\text { RR, SDRR, RMSSD, } \\
\text { LFun*, HFun*, LF/ } \\
\text { HF*, TP(ms²) e } \\
\text { Entropia de Sample. }\end{array}$ & $\begin{array}{l}\text { Valores inferiores de HF e maiores valores } \\
\text { de LF e a relação LF/HF foram observados } \\
\text { em crianças obesas ao serem comparadas } \\
\text { a crianças não obesas. Esses dados refle- } \\
\text { tem uma disfunção no SNA dessa popula- } \\
\text { ção. }\end{array}$ \\
\hline $\begin{array}{l}\text { Kaufman et al., } \\
2008 .\end{array}$ & $\begin{array}{l}\text { Crianças obesas }(\mathrm{N}=15) \text { : Média } \\
\text { de idade de } 11,4 \pm 0,5 \text { anos e } \\
\text { classificação da obesidade de } \\
\text { acordo com o IMC correspondente } \\
\text { ao percentil } 85 \text {. }\end{array}$ & $\begin{array}{l}\text { Avaliar os efeitos de } 5 \text { me- } \\
\text { ses de reeducação alimen- } \\
\text { tar sobre o SNA e o sistema } \\
\text { endotelial de crianças obe- } \\
\text { sas. }\end{array}$ & $\begin{array}{l}\text { SDNN, RMSSD**, } \\
\text { LFun, HFun e LF/ } \\
\text { HF**. }\end{array}$ & $\begin{array}{l}\text { Os resultados refletem um aumento do } \\
\text { SNP. Portanto pode-se concluir que a dieta } \\
\text { alimentar contribui para o controle do peso } \\
\text { e da disfunção do SNA. }\end{array}$ \\
\hline $\begin{array}{l}\text { Ancona et al., } \\
2009 .\end{array}$ & $\begin{array}{l}\text { Grupo obeso }(\mathrm{N}=15) \text { : Média de } \\
\text { idade } 9,6 \pm 0,7 \text { anos e IMC } 23,2 \\
\pm 0,8 \mathrm{Kg} / \mathrm{m}^{2} \text {. } \\
\text { Grupo não obeso }(\mathrm{N}=15) \text { : Média } \\
\text { de idade } 9,4 \pm 0,6 \text { anos e IMC } \\
16,3 \pm 2,2 \mathrm{Kg} / \mathrm{m}^{2} \text {. }\end{array}$ & $\begin{array}{l}\text { Analisar possíveis alterações } \\
\text { na modulação vagossimpá- } \\
\text { tica cardíaca de crianças } \\
\text { obesas e eutróficas nas po- } \\
\text { sições supina e bípede. }\end{array}$ & $\begin{array}{l}\text { Intervalo RR médio, } \\
\text { Desvio-padrão dos } \\
\text { intervalos RR, } \\
\text { RMSSD, pNN50, } \\
\mathrm{HF}\left(\mathrm{ms}^{2}\right), \mathrm{LH}\left(\mathrm{ms}^{2}\right) \text { e } \\
\mathrm{LF} / \mathrm{HF} \text {. }\end{array}$ & $\begin{array}{l}\text { Tanto na posição supina quanto na posição } \\
\text { bípede, não houve diferenças significativas } \\
\text { na modulação autonômica cardíaca entre } \\
\text { os grupos, sugerindo assim que a modula- } \\
\text { ção autonômica cardíaca não sofre influên- } \\
\text { cias desse grau de obesidade infantil. }\end{array}$ \\
\hline $\begin{array}{l}\text { Paschoal et al, } \\
2009 .\end{array}$ & $\begin{array}{l}\text { Grupo obeso ( } \mathrm{N}=15) \text { : Média de } \\
\text { idade } 10,2 \pm 0,7 \text { anos e IMC } 23,9 \\
\pm 1 \mathrm{Kg} / \mathrm{m}^{2} \text {. } \\
\text { Grupo não obeso }(\mathrm{N}=15) \text { : Média } \\
\text { de idade } 9,8 \pm 0,7 \text { anos e IMC } \\
17,7 \pm 1,6 \mathrm{~kg} / \mathrm{m}^{2} \text {. }\end{array}$ & $\begin{array}{l}\text { Adicionar os mais relevan- } \\
\text { tes e recorrentes paradig- } \\
\text { mas da influência precoce da } \\
\text { obesidade em futuras doen- } \\
\text { ças cardiovasculares e me- } \\
\text { tabólicas. }\end{array}$ & $\begin{array}{l}\text { Média dos intervalos } \\
\text { RR, SDNN, RMSSD, } \\
\text { pNN50, LFun*, } \\
\text { HFun, LF(ms²), } \\
\text { HF }\left(\mathrm{ms}^{2}\right) \text {, LF/HF*. }\end{array}$ & $\begin{array}{l}\text { O grupo obeso apresentou maior atuação } \\
\text { do SNS devido aos maiores valores dos ín- } \\
\text { dices LFun e LF/HF em relação ao grupo } \\
\text { não obeso. }\end{array}$ \\
\hline $\begin{array}{l}\text { Dipla et al., } \\
2010 .\end{array}$ & $\begin{array}{l}\text { Grupo obeso }(\mathrm{N}=14) \text { : Média de } \\
\text { idade } 11,7 \pm 0,23 \text { anos e IMC } \\
29,2 \pm 0,9 \mathrm{Kg} / \mathrm{m}^{2} \text {. } \\
\text { Grupo não obeso }(\mathrm{N}=13) \text { : Média } \\
\text { de idade } 11,8 \pm 0,22 \text { anos e IMC } \\
18,9 \pm 0,3 \mathrm{Kg} / \mathrm{m}^{2} \text {. }\end{array}$ & $\begin{array}{l}\text { Testar a hipótese de que } \\
\text { crianças obesas apresentam } \\
\text { respostas hemodinâmicas e } \\
\text { controle barorreflexo dife- } \\
\text { rentes a crianças não obe- } \\
\text { sas frente a exercício } \\
\text { isométrico e recuperação. }\end{array}$ & $\begin{array}{l}\text { RMSSD*, SD } 1 *, \text { SD2 } \\
\text { e SD1/SD2. }\end{array}$ & $\begin{array}{l}\text { Durante o exercício isométrico, as crian- } \\
\text { ças obesas apresentam menor atuação do } \\
\text { SNP comparado com as crianças não obe- } \\
\text { sas. Já no período de recuperação não hou- } \\
\text { ve diferença entre os grupos. }\end{array}$ \\
\hline
\end{tabular}




\begin{tabular}{|c|c|c|c|c|}
\hline $\begin{array}{l}\text { Autores e ano } \\
\text { de publicação }\end{array}$ & Características da população & Objetivos & Índices avaliados & Conclusões \\
\hline $\begin{array}{l}\text { Vanderlei et } \\
\text { al., } 2010 .\end{array}$ & $\begin{array}{l}\text { Grupo obeso }(\mathrm{N}=51) \text { : Média de } \\
\text { idade } 10,06 \pm 1,27 \text { anos, IMC } \\
28,45 \pm 3,54 \mathrm{Kg} / \mathrm{m}^{2} \text {. } \\
\text { Grupo não obeso }(\mathrm{N}=61) \text { : Média } \\
\text { de idade } 10,49 \pm 1,39 \text { anos, IMC } \\
17,42 \pm 1,76 \mathrm{Kg} / \mathrm{m}^{2} \text {. }\end{array}$ & $\begin{array}{l}\text { Investigar a dinâmica da fre- } \\
\text { quência cardíaca de crianças } \\
\text { obesas por meio dos índices } \\
\text { de longo e curto prazo atra- } \\
\text { vés do expoente fractal DFA, } \\
\text { juntamente a análise da VFC } \\
\text { no domínio da frequência. }\end{array}$ & $\begin{array}{l}\text { LFun, HFun, } \\
\left.\text { LF(ms }{ }^{2}\right)^{*} \\
\operatorname{HF}\left(m s^{2}\right)^{*}, \mathrm{LF} / \mathrm{HF}, \\
\text { Alfa1*, Alfa2 e } \\
\text { relação Alfa1/Alfa2 }\end{array}$ & $\begin{array}{l}\text { O grupo obeso apresentou disfunção auto- } \\
\text { nômica, caracterizada pela redução de ati- } \\
\text { vidade do SNS e SNP e perda da correla- } \\
\text { ção fractal de curto prazo da FC. }\end{array}$ \\
\hline $\begin{array}{l}\text { Vanderlei et } \\
\text { al., } 2010 .\end{array}$ & $\begin{array}{l}\text { Grupo obeso }(\mathrm{N}=61) \text { : Média de } \\
\text { idade } 10,20 \pm 1,47 \text { anos e IMC } \\
29,30 \pm 4,74 \mathrm{Kg} / \mathrm{m}^{2} \text {. } \\
\text { Grupo não obeso }(\mathrm{N}=72) \text { : Média } \\
\text { de idade } 10,57 \pm 1,51 \text { anos e IMC } \\
17,37 \pm 1,82 \mathrm{Kg} / \mathrm{m}^{2} \text {. }\end{array}$ & $\begin{array}{l}\text { Investigar a modulação au- } \\
\text { tonômica de crianças obesas } \\
\text { e eutróficas por meio de ín- } \\
\text { dices da VFC obtidos por } \\
\text { métodos geométricos. }\end{array}$ & $\begin{array}{l}\text { RRtri*, TINN*, } \\
\text { SD1*, SD2*, SD1/ } \\
\text { SD2 e análise visual } \\
\text { do plot de Poincaré. }\end{array}$ & $\begin{array}{l}\text { Nas crianças obesas analisadas ocorreram } \\
\text { reduções dos índices geométricos, suge- } \\
\text { rindo que, nestas crianças, a VFC global } \\
\text { está reduzida assim como a atividade } \\
\text { parassimpática. }\end{array}$ \\
\hline $\begin{array}{l}\text { Vanderlei et } \\
\text { al., } 2010 .\end{array}$ & $\begin{array}{l}\text { Grupo obeso }(\mathrm{N}=56) \text { : Média de } \\
\text { idade } 9,96 \pm 1,31 \text { anos e IMC } \\
28,58 \pm 3,66 \mathrm{Kg} / \mathrm{m}^{2} \text {. } \\
\text { Grupo não obeso }(\mathrm{N}=65) \text { : Média } \\
\text { de idade } 10,39 \pm 1,42 \text { anos e IMC } \\
17,33 \pm 1,77 \mathrm{~kg} / \mathrm{m}^{2} \text {. }\end{array}$ & $\begin{array}{l}\text { Comparar a função autonô- } \\
\text { mica de crianças obesas e } \\
\text { eutróficas por meio da aná- } \\
\text { lise da VFC. }\end{array}$ & $\begin{array}{l}\text { RMSSD*, SDNN*, } \\
\text { pNN50*, SD } 1 *, \\
\text { SD2*, SD } 1 / S D 2, \\
\text { LFun, HFun, } \\
\text { LF }\left(\mathrm{ms}^{2}\right)^{*}, \\
\mathrm{HF}\left(\mathrm{ms}^{2}\right)^{*}, \mathrm{LF} / \mathrm{HF} .\end{array}$ & $\begin{array}{l}\text { Os resultados sugerem uma diminuição na } \\
\text { atividade do SNS e SNP de crianças obe- } \\
\text { sas devido aos valores encontrados dos ín- } \\
\text { dices ao serem comparados a crianças } \\
\text { eutróficas. }\end{array}$ \\
\hline $\begin{array}{c}\text { Sekine et al., } \\
2011 .\end{array}$ & $\begin{array}{l}\text { Grupo obeso }(N=7) \text { : Média de } \\
\text { idade } 9,21 \pm 0,33 \text { anos e IMC } \\
21,6 \pm 0,75 \mathrm{Kg} / \mathrm{m}^{2} \text {. } \\
\text { Grupo não obeso }(\mathrm{N}=9) \text { : Média } \\
\text { de idade } 9,11 \pm 0,29 \text { anos e IMC } \\
16,2 \pm 1,32 \mathrm{~kg} / \mathrm{m}^{2} \text {. }\end{array}$ & $\begin{array}{l}\text { Determinar a relação entre } \\
\text { obesidade e atividade do } \\
\text { SNA em crianças saudáveis. }\end{array}$ & $\begin{array}{l}\text { SD, CV, RMSSD, } \\
\operatorname{TP}\left(\mathrm{ms}^{2}\right)^{*}, \\
\operatorname{VLF}\left(\mathrm{ms}^{2}\right)^{*}, \operatorname{LF}\left(\mathrm{ms}^{2}\right), \\
\mathrm{HF}\left(\mathrm{ms}^{2}\right), \operatorname{LFun} \text {, } \\
\text { HFun* e LF/HF*. }\end{array}$ & $\begin{array}{l}\text { De acordo com os valores obtidos dos ín- } \\
\text { dices e sua comparação entre os grupos } \\
\text { as crianças obesas apresentam maior atua- } \\
\text { ção da atividade simpática enquanto que a } \\
\text { atividade parassimpática encontra-se dimi- } \\
\text { nuída. }\end{array}$ \\
\hline
\end{tabular}

* Diferença estática entre o grupo obeso e não obeso; **Diferença estatística entre antes e depois da intervenção; Abreviaturas: $\mathrm{N}=$ número de crianças analisadas; IMC = índice de massa corporal; SNA =sistema nervoso autônomo; SNS = sistema nervoso simpático; SNP = sistema nervoso parassimpático; VFC = variabilidade da frequência cardíaca; DFA = análise de flutuação depurada de tendência; RMSSD = raiz quadrada da média do quadrado das diferenças entre intervalos RR normais adjacentes; SD1 = desvio-padrão da variabilidade instantânea batimento-a-batimento; SD2 = desvio-padrão a longo prazo dos intervalos RR contínuos; SD1/SD2 = razão entre as variações curta e longa dos intervalos RR; SDNN = desvio padrão de todos os intervalos RR normais; LF = componente de baixa frequência; HF = componente de alta frequência; LF/HF = razão entre os componentes de baixa e alta frequência; un = unidade normalizada; $\mathrm{ms}^{2}=$ milissegundos quadrado; $\mathrm{TP}=$ potência total em $\mathrm{ms}^{2} ;$ Alfa 1 = expoente fractal de curto prazo; Alfa $2=$ expoente fractal de longo prazo; Alfa1/Alfa2 = razão entre expoentes fractais de curto e longo prazo; pNN50 = porcentagem dos intervalos RR adjacentes com diferença de duração maior que $50 \mathrm{~ms}$; RRtri = índice triangular; TINN = interpolação triangular dos intervalos RR; SDRR = desvio padrão dos intervalos RR normais; SD = desvio padrão dos intervalos RR normais; CV = coeficiente de variação; VLF = componente de muito baixa frequência.

\section{DISCUSSÃo}

A análise dos textos selecionados demonstrou que a obesidade em crianças de até 12 anos de idade promove alterações no SNA que podem ser assim caracterizadas: 1) em geral crianças obesas apresentam redução da atividade do SNA parassimpático (SNP) em comparação a crianças não obesas; 2) os resultados em relação ao SNA simpático (SNS) são divergentes, com estudos apontando atividade simpática reduzida, outros mostrando aumento e ainda estudos que não encontraram alterações no SNS em crianças obesas em comparação a não obesas; 3) intervenções como redução do peso devido à dieta alimentar e atividade física são capazes de promover melhoras no SNA dessa população.

Alterações tanto da atividade do componente simpático quanto parassimpático do SNA foram descritas em crianças obesas por diversos autores. Nagai et al ${ }^{26}$ investigaram as alterações do SNA, por meio dos índices LF, HF e TP, em crianças obesas e correlacionaram a duração da obesidade com o grau de disfunção do SNA. Os autores observaram que crianças obesas apresentam disfunção tanto do componente simpático quanto parassimpáti- co, caracterizada por redução de todos os índices de VFC analisados, quando comparadas a crianças não obesas, e discutem que a redução da atividade do SNP pode estar associada a futuras neuropatias autonômicas, disfunções metabólicas e altos níveis de pressão arterial, enquanto que, a redução da atividade do SNS, sugere que seja verdadeira a hipótese de que a diminuição da atividade simpática promova menor gasto energético e, consequentemente, um desequilíbrio energético positivo que leva ao quadro de obesidade. Correlação negativa entre a duração da obesidade com o grau de disfunção do SNA foi observada, indicando que quanto menor a atividade do SNA maior a duração da obesidade.

Este mesmo padrão de comportamento do SNA foi descrito no estudo realizado por Vanderlei et $a^{30}$, o qual avaliou em crianças obesas índices de VFC nos domínios do tempo (RMSSD, pNN50 e SDNN), frequência (LF e HF, em unidades normalizadas (un) e $\mathrm{ms}^{2}$, e a relação LF/HF) e pelo plot de Poincaré (SD1, SD2 e relação SD1/SD2). Foi observada redução de todos esses índices em crianças obesas, indicando diminuição da variabilidade global e da atividade tanto do SNP quanto SNS. 
Reduções da atividade do SNP e da variabilidade global foram também observadas em crianças obesas por Vanderlei et al ${ }^{28}$ por meio da análise de índices geométricos de VFC. Utilizando os índices RRtri, TINN, SD1, SD2, relação SD1/SD2 e a análise visual do plot de Poincaré, os autores mostraram que crianças obesas apresentam redução na atividade parassimpática, caracterizada por diminuição do índice SD1, associada à redução da atividade global do SNA, observada pela análise dos índices RRtri, TINN, SD2 e análise visual do plot de Poincaré.

Analisando a atividade autonômica por meio dos índices RMSSD, SD1, SD2 e da relação SD1/ SD2 frente ao exercício isométrico de flexão de cotovelo e durante sua recuperação, Dipla et al ${ }^{27}$ não observaram alterações significantes do SNA entre crianças obesas e não obesas no período de recuperação, porém, durante a realização do exercício, crianças obesas apresentaram menores valores dos índices RMSSD e SD1, indicando uma menor atuação do SNP nessas crianças. Segundo os autores, o fato dessa alteração somente aparecer durante o exercício pode estar relacionada a uma fase precoce da obesidade, mostrando que a realização do exercício evidencia alterações que não são observadas em repouso.

Um estudo realizado por Paschoal et al $^{2}$ apontou um comportamento diferente do observado acima para o SNS. Avaliando diferentes índices de VFC em crianças obesas e não obesas na posição ortostática, os autores observaram que apenas os índices LFun e a relação LF/HF apresentaram diferenças significantes, com valores maiores para crianças obesas, indicando que elas apresentam hiperatividade do SNS. Os autores relataram que as crianças abordadas em seu estudo apresentam uma faixa etária diferente dos grupos estudados em outros ensaios clínicos, o que poderia ser um dos fatores responsáveis pelos resultados divergentes em comparação a outros estudos.

Outros autores também confirmam esse padrão de comportamento do SNS. Sekine et al ${ }^{31}$ observaram em crianças obesas maiores valores do índice LFun e da relação LF/HF em comparação ao grupo não obeso, o que indica maior atividade simpática nessas crianças. Além disso, índices que refletem a atuação do SNP (TP (ms ${ }^{2}$ ) e HFun), apresentaram valores menores para o grupo obeso, sugerindo que essas crianças apresentam diminuição da atividade parassimpática.

Mesmo padrão de comportamento do SNA foi relatado no ensaio clínico de Kaufman et al ${ }^{32}$, os quais examinaram a função autonômica cardiovascular e sua potencial relação com resistência à leptina, resistência à insulina, estresse oxidativo e inflamatório em crianças com diferentes níveis de obesidade. Os autores observaram menores valores de HFun e maiores valores de LFun e da relação LF/HF nas crianças obesas em comparação as não obesas. Nesse estudo não foram observadas alterações entre o grupo de crianças acima do peso em comparação ao obeso, o que indica que a atuação do SNA não se altera até a obesidade ser substancialmente aumentada.

Diferentemente dos estudos acima descritos, Ancona et al ${ }^{34}$ não observaram diferenças significantes entre crianças obesas e não obesas, nas posições supina e bípede, por meio dos índices SDNN, RMSSD, pNN50, $\mathrm{HF}\left(\mathrm{ms}^{2}\right), \mathrm{LH}\left(\mathrm{ms}^{2}\right)$, relação LF/HF e da média dos intervalos RR, sugerindo que o grau de obesidade apresentado pelas crianças obesas analisadas nesse estudo não foi capaz de promover alterações no SNA.

O único estudo encontrado que avaliou a VFC em crianças obesas por meio de métodos não lineares foi publicado por Vanderlei et $\mathrm{al}^{8}$. Utilizando a análise de flutuação depurada sem tendência (Detrended Fluctuation Analysis - DFA), os autores relataram que crianças obesas apresentam perda das propriedades de correlação fractal de curto prazo da dinâmica da frequência cardíaca, em direção a uma dinâmica mais aleatória, associada à redução da atividade tanto do SNS quanto SNP. Segundo os autores, a perda precoce da dinâmica fractal da frequência cardíaca está associada ao maior risco de desenvolvimento de doenças cardiovasculares.

Os estudos descritos apontam alguns resultados divergentes em relação ao comportamento dos componentes simpático e parassimpático do SNA em crianças obesas. Uma das possíveis causas para essas divergências é a dificuldade em se controlar variáveis que estão presentes nos grupos estudados como: idade, sexo, histórico de obesidade, dieta e hábitos alimentares individuais, níveis de atividade física e estresse emocional 8, 26, 29, 31,35 . Além disso, as limitações metodológicas para avaliação da atividade do SNS, em função dos índices LF e da relação LF/HF não serem índices puros da atividade deste componente do SNA 26, 31, podem também ter contribuído para esses resultados conflitantes.

A literatura mostra ainda que a redução do peso corporal e a atividade física são capazes de promover melhoras no SNA de crianças obesas. Avaliando os efeitos de cinco meses de reeducação alimentar sobre o SNA e o sistema endotelial de crianças obesas, Kaufman et al ${ }^{33}$ observaram além de menores valores de gordura corporal, peso, IMC e percentual de gordura, diminuição da relação LF/ HF e aumento do índice RMSSD, indicando que o programa de reeducação alimentar promoveu aumento da atividade parassimpática. Os autores apontam que a redução na formação de radicais livres, aumento da atividade do receptor de leptina e diminuição da leptina decorrentes da perda de peso, podem estar relacionados ao aumento da atividade do SNP nessas crianças.

Em relação à atividade física, Nagai et al ${ }^{29}$ avaliaram índices de VFC (LF, HF e TP em $\mathrm{ms}^{2}$ ) de crianças obesas e não obesas ativas e inativas, para verificar se o SNA está alterado devido à obesidade e à inatividade física. As comparações entre crianças obesas e não obesas com níveis de atividade 
física semelhantes mostraram que as crianças obesas, independentemente do nível, apresentaram menores valores dos índices avaliados. Já as comparações entre as crianças obesas mostraram que as mais ativas apresentaram valores do índice TP maiores que as sedentárias, indicando que a atividade física pode diminuir as alterações induzidas pela obesidade no SNA de crianças obesas.

Nesse contexto, deve ser ressaltado que intervenções com dieta e exercício físico, as quais

\section{REFERÊNCIAS}

1. Yakinci C, Mungen B, Karabiber H, Tayfun M, Evereklioglu C. Autonomic nervous system functions in obese children. Brain Dev. 2000;22(3):151-3.

2. Paschoal MA, Trevizan PF, Scodeler NF. Heart rate variability, blood lipids and physical capacity of obese and non-obese children. Arq Bras Cardiol. 2009;93(3):223-9.

3. Oliveira AMA, Cerqueira EMM, Souza JS, Oliveira AC. Sobrepeso e obesidade infantil: influência de fatores biológicos e ambientais em Feira de Santana, BA. Arq Bras Endocrinol Metab. 2003;47(2):144-50.

4. Low S, Chin CM, Deurenberg-Yap M. Review on epidemic of obesity. Ann Acad Med. 2009; 38: 57-65.

5. Enes CC, Slater B. Obesidade na adolescência e seus principais fatores determinantes. Rev Bras Epidemiol. 2010;13(1):163-71.

6. Yosipovitch G, DeVore A, Dawn A. Obesity and the skin: Skin physiology and skin manifestations of obesity. J Am Acad Dermatol. 2007;56(6):901-16.

7. Simon VGN, Souza JMP, Leone C, Souza SB. Prevalência de sobrepeso e obesidade em crianças de dois a seis anos matriculadas em escolas particulares no município de São Paulo. Rev Bras Crescimento Desenvolvimento Hum. 2009;19(2): 211-18.

8. Vanderlei LCM, Pastre CM, Júnior IFF, de Godoy MF. Fractal correlation of heart rate variability in obese children. Auton Neurosci. 2010;155(12): 125-9.

9. Rabbone I, Bobbio A, Rabbia F, Bertello M, Ignaccolo M, Saglio E, et al. Early cardiovascular autonomic dysfunction, b cell function and insulin resistance in obese adolescents. Acta Bio Medica. 2009;80:29-35.

10. Junior RDRL, Cardoso-Demartini AA, Ono AHA, Andrade GC. Prevalência de obesidade em crianças e adolescentes com diabetes melito tipo 1. Rev Paul Pediatr. 2008;26(2):142-5.

11. Brunetto ANF, Roseguini BT, Silva BM, Hirai DML, Guedes DP. Respostas autonômicas cardíacas à manobra de tilt em adolescentes obesos. Rev Assoc Med Bras. 2005;51:256-60. buscam reduzir o grau de obesidade, podem ser capazes de amenizar as alterações observadas no SNA de crianças obesas e devem ser estimuladas precocemente para amenizar ou eliminar os riscos produzidos pela obesidade.

Os trabalhos demonstraram que a obesidade produz modificações no comportamento do SNA em crianças obesas. Em geral, observa-se redução da atividade do SNP, porém os resultados em relação à atividade simpática são inconclusivos.

12. Rabbia F, Silke B, Conterno A, Grosso T, De Vito $B$, Rabbone I, et al. Assessment of cardiac autonomic modulation during adolescent obesity. Obes res. 2003;11(4):541-8.

13. Lazarova Z, Tonhajzerova I, Trunkvalterova Z, Brozmanova A, HonzíKová N, Javorka K, et al. Baroreflex sensitivity is reduced in obese normotensive children and adolescents. Can J Physiol Pharmacol. 2009;87(7): 565-71.

14. Tonhajzerova I, Javorka M, Trunkvalterova Z, Chroma O, Javorkova J, Lazarova Z, et al. Cardio-respiratory interaction and autonomic dysfunction in obesity. J Physiol Phamarcol. 2008;59(6):709-18.

15. Molfino A, Fiorentini A, Tubani L, Martuscelli M, Fanelli FR, Laviano A. Body mass index is related to autonomic nervous system activity as measured by heart rate variability. Eur J Clin Nutr. 2009;63(10):1263-5.

16. Ancona MC, Scodeler NF, Guidi RM, Paschoal MA. Variabilidade de frequência cardíaca em crianças eutróficas e obesas nas posições supina e bípede. Rev Cienc Med. 2009;18(2):69-79.

17. Wu JS, Lu FH, Yang YC, Lin TS, Huang YH, Wu $\mathrm{CH}$, et al. Epidemiological evidence of altered cardiac autonomic function in overweight but not underweight subjects. Int J Obes. 2008;32(5): 788-94.

18. Emdin M, Gastaldelli A, Muscelli E, Macerata A, Natali A, Camastra S, et al. Hyperinsulinemia and Autonomic Nervous System Dysfunction in Obesity : Effects of Weight Loss. Circulation. 2001;103(4):513-9.

19. Schmid K, Schönlebe J, Drexler H, MueckWeymann M. Associations between being overweight, variability in heart rate, and wellbeing in the young men. Cardiol Young. 2010;20(1):54-9.

20. Vanderlei LCM, Pastre CM, Hoshi RA, Carvalho TD, Godoy MF. Noções básicas de variabilidade da frequência cardíaca e sua aplicabilidade clínica. Rev Bras Cir Cardiovasc. 2009;24(2):205-17.

21. Manzano BM, Vanderlei LCM, Ramos EM, Ramos D. Efeitos Agudos do Tabagismo sobre a Modulação Autonômica: Análise por Meio do Plot de Poincaré. Arq Bras Cardiol. 2011;96(2):154-60 
22. Acharya RU, Kannathal N, Sing O, Ping L, Chua T. Heart rate analysis in normal subjects of various age groups. Biomed Eng Online. $2004 ; 3: 24$.

23. Lambert GW, Straznicky NE, Lambert EA, Dixon JB, Schlaich MP. Sympathetic nervous activation in obesity and the metabolic syndrome Causes, consequences and therapeutic implications. Pharmacol Ther. 2010;126(2):159-72.

24. Masuo K, Lambert GW. Relationships of adrenoceptor polymorphisms with obesity. J Obes. 2011;1-10.

25. Avsar A, Acarturk G, Melek M, Kilit C, Celik A, Onrat E. cardiac autonomic function evaluated by the heart rate turbulence method was not changed in obese patients without comorbidities. J Korean Med Sci. 2007; 22: 62932.

26. Nagai N, Matsumoto T, Kita H, Moritani T. Autonomic nervous system activity and the state and development of obesity in Japanese school children. Obes Res. 2003;11(1):25-32.

27. Dipla K, Zafeiridis A, Koidou I, Geladas N, Vrabas IS. Altered hemodynamic regulation and reflex control during exercise and recovery in obese boys. Am J Physiol. 2010; 299(6): H2090-6.

28. Vanderlei LC, Pastre CM, Freitas Júnior IF, Godoy MF. Geometric indexes of heart rate variability in obese and eutrophic children. Arq Bras Cardiol. 2010;95(1):35-40.
29. Nagai N, Moritani T. Effect of physical activity on autonomic nervous system function in lean and obese children. Int J Obes Relat Metab Disord. 2004;28(1):27-33.

30. Vanderlei LC, Pastre CM, Freitas Júnior IF, Godoy MF. Analysis of cardiac autonomic modulation in obese and eutrophic children. Clinics. 2010;65(8):789-92.

31. Sekine M, Izumi I, Yamagami T, Kagamimori S. Obesity and cardiac autonomic nerve activity in healthy children: Results of the toyama birth cohort study. Environ Health Prev Med. 2001;6(3):149-53.

32. Kaufman $C L$, Kaiser DR, Steinberger J, Kelly AS, Dengel DR. Relationships of cardiac autonomic function with metabolic abnormalities in childhood obesity. Obesity. 2007;15(5):1164-71.

33. Kaufman C, Kaiser D, Kelly A, Dengel J, Steinberger J, Dengel D. Diet revision in overweight children: effect on autonomic and vascular function. Clin Auton Res. 2008;18:105-8.

34. Ancona MC, Scodeler NF, Guidi RM, Paschoal MA. Variabilidade de frequência cardíaca em crianças eutróficas e obesas nas posições supina e bípede. Rev Cienc Med. 2009;18(2):69-79.

35. Krishnan B, Jeffery A, Metcalf B, Hosking J, Voss $\mathrm{L}$, Wilkin $\mathrm{T}$, et al. Gender differences in the relationship between heart rate control and adiposity in young children: a cross-sectional study (EarlyBird 33). Pediatric Diabetes. 2009;10(2):127-34. 